\title{
Pump-Induced Wavefront Distortion in Prototypical NIF/LMJ Amplifiers - Modeling and Comparison with Experiments
}

\author{
M. Rotter, K. Jancaitis, C. Marshall, \\ L. Zapate, A. Erlandson, G. LeTouzé, \\ and S. Seznec
}

This paper was prepared for submittal to the Third Annual International Conference on Solid State Lasers for Application (SSLA) to Inertial Confinement Fusion (ICF) Monterey, California June 7-12, 1998

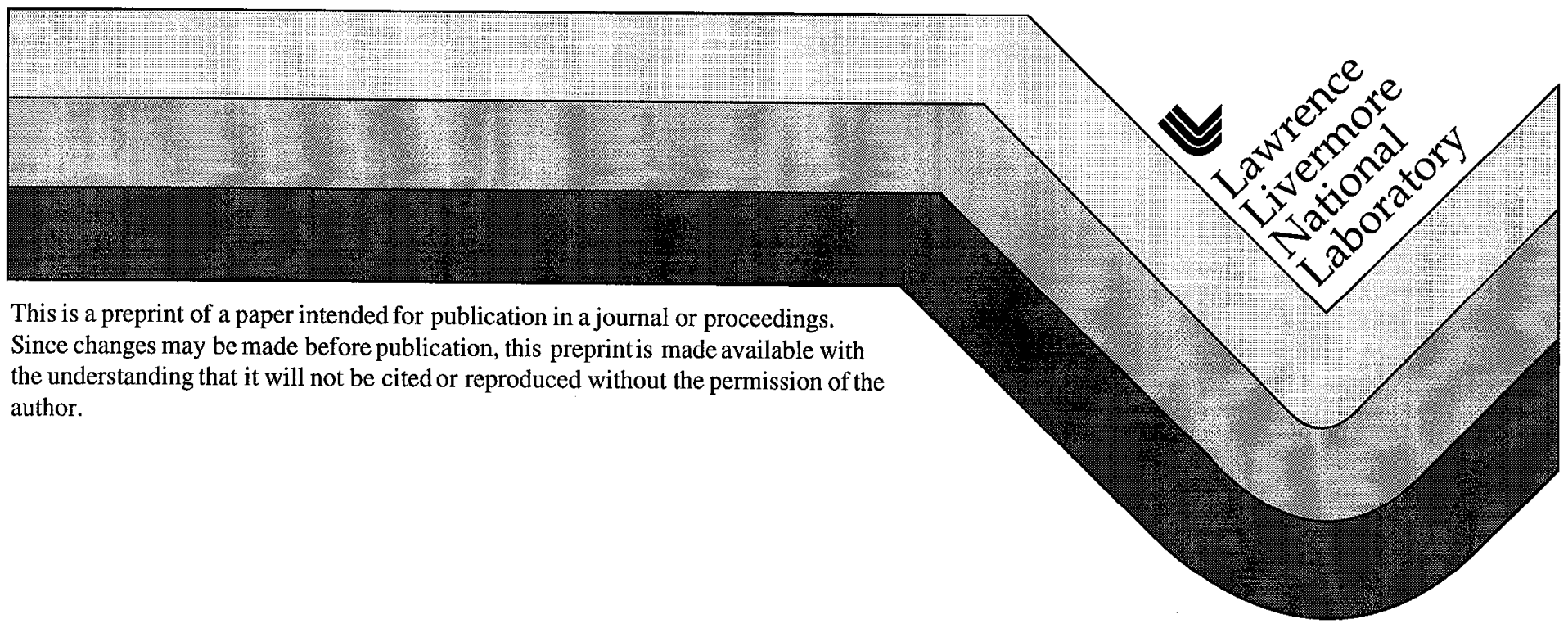




\section{DISCLAIMER}

This document was prepared as an account of work sponsored by an agency of the United States Government. Neither the United States Government nor the University of California nor any of their employees, makes any warranty, express or implied, or assumes any legal liability or responsibility for the accuracy, completeness, or usefulness of any information, apparatus, product, or process disclosed, or represents that its use would not infringe privately owned rights. Reference herein to any specific commercial product, process, or service by trade name, trademark, manufacturer, or otherwise, does not necessarily constitute or imply its endorsement, recommendation, or favoring by the United States Government or the University of California. The views and opinions of authors expressed herein do not necessarily state or reflect those of the United States Government or the University of Califurnia, and shall not be used for advertising or product endorsement purposes. 


\author{
Pump-Induced Wavefront Distortion in Prototypical NIF/LMJ Amplifiers - \\ Modeling and Comparison with Experiments* \\ Mark Rotter, Ken Jancaitis, Chris Marshall, Luis Zapata and Al Erlandson \\ Lawrence Livermore National Laboratory \\ P.O. Box $5504 \mathrm{~L}-477$ \\ Livermore, CA. 94551 \\ Geoffroy LeTouzé and Stephane Seznec \\ Commissariat à l'Energie Atomique \\ Centre d'Etudes de Limeil-Valenton \\ Boite Postale 27 \\ Villeneuve, St. Georges, FRANCE 94190
}

\begin{abstract}
In large-aperture laser amplifiers such as those envisioned for the National Ignition Facility (NIF) and Laser Megajoules (LMJ) lasers, the geometry is such that the front and back faces of the laser slab are heated unevenly by the pump process. This uneven heting results in a mechanical deformation of the laser slab and consequent internal stresses. The deformation and stresses, along with a temperature-dependent refractive index variation, result in phase variations across the laser beam (so-called pump-induced wavefront distortions). These phase variations lead to beam steering which may affect frequency conversion as well as energy-on-target. We have developed a model which allows us to estimate the pump-induced wavefront distortion for a given amplifier configuration as well as the spatially-resolved depolarization. The model is compared with experiments taken in our amplifier development laboratory, AMPLAB.
\end{abstract}

\title{
1. Introduction
}

We are currently developing large-aperture amplifiers for the National Ignition Facility (NIF) and Laser Megajoules (LMJ) lasers. These multi-segment amplifiers are of the flashlamp-pumped, $\mathrm{Nd}$ :Glass type and are designed to propagate a nominally $36 \mathrm{~cm}$ square beam. The apertures within a particular amplifier bundle are arranged in a four-high by two-wide configuration and utilize two side flashlamp arrays and a central flashlamp array for pumping, see Fig. 1. As shown in the figure, the slabs are oriented at Brewster's angle and are pumped on both sides by arrays of flashlamps, denoted as central arrays (lamps which pump both slabs) and side arrays. The geometry of the amplifier results in one end of the slab situated closer to the lamps than the other side.

Consequently, the amount of heat deposited in the slab (primarily from the quantum defect of the broad-band pump light) is uneven front-to-back as well as side-to-side.

This uneven pumping results in a warping of the laser slab, indicated by the dashed lines in Fig. 1. As a result, an initially plane wavefront incident on such a slab will not remain planar upon exit. Ultimately, wavefront distortion is due to differences in the optical path (defined as the refractive index times the physical path length) at one point in the aperture $v s$ another. We have seen one source of these optical path differences (or OPDs) - namely the mechanical distortion of the laser slab. There is another source of

\footnotetext{
* This research was perfomred under the auspices of the U.S. Department of Energy by Lawrence Livermore National Laboratory under contract number W-7405-ENG-48.
} 
OPD, namely the spatially-varying refractive index to which temperature and sress contribute.

We have developed a model which takes all of the above effects into account and which allows us to predict the pump-induced wavefront distortion for these large-aperture amplifiers. In this paper, will describe various aspects of the model and present comparisons between the model and experimental data taken on AMPLAB, our amplifier test laboratory.

\section{Pump-induced-wavefront-distortion model}

\subsection{Description of model}

As mentioned in the introduction, the non-uniform deposition of heat in the laser slab is responsible for slab distortions and aberrations of any plane wavefront incident on it. Thus, the fundamental ideas may be summarized as follows: The non-uniform heat deposition results in a distortion of the laser slab and accompanying stresses. The distortion of the laser slab, in conjunction with the temperature and stress-induced refractive index variations result in the OPDs and consequent wavefront distortion.

In order to calculate the various effects listed above, we use a suite of computer codes: TOPAZ3D ${ }^{1}$ to calculate the temperature distribution within the laser slab, NIKE3D ${ }^{2}$ to calculate the displacements and stresses from the temperature field given by TOPAZ3D, and OPL, which calculates the OPDs given the results from NIKE3D. The codes NIKE3D and TOPAZ3D are 3-dimensional finite element analysis codes which have been in use at LLNL for over ten years. The optics code OPL is an in-house code based on the BREW code developed by Said Doss and Robert Gelinas. ${ }^{3}$

\subsection{Determination of temperature}

The first step in calculating the wavefront distortion is to determine the temperature distribution within the laser slab. In order to do this, we need to specify the thermal source function as a function of position and time. In general, this thermal source function is an arbitrary function of position and time. For purposes of this model, however, we have assumed a separable source function, i.e.:

$$
Q(x, y, z, t)=A s(x)\left[g_{0}(y) f(z)+g_{h}(y) f(h-z)\right] u(t)+Q_{e c}
$$

where $s(x)$ denotes the vertical variation of the pump profile, $g_{0, k}(y)$ denotes the horizontal variation of the pump profile at $z=0, h$ respectively, $f(z)$ denotes the pump profile through the thickness of the slab, and $Q_{e c}$ is the thermal source term for the edge cladding. The units for this source function are W/cm $\mathrm{cm}^{3}$. In Eq. (1), $h$ is the thickness of the slab and $A$ is a constant multiplier. Each of these terms will now be described.

In the multisegment amplifiers envisioned for use in the NIF, there is strong vertical symmetry. The flashlamps are oriented vertically, and there are silver-coated metal reflectors at the top and bottom of the pump cavity. As a result, we have taken the function $s(x)$ to be a constant. In reality, the reflectors are not perfect and so there is a slight roll-off in pump light at the extreme top and bottom of the pump cavity. We have found that this is a small effect insofar as calculating the pump-induced wavefront distortion is concemed, and so have elected to keep $s(x)$ a constant.

As mentioned above, the geometry of the pump cavity results in a roll-off of the pump radiation from one side of the aperture to the other. We used our 2-D+ ray-trace $\operatorname{code}^{4}$ to claculate the distribution of pump light across the laser slab. An example of the 
functions so obtained is shown in Fig. 2. Because these functions can be rather convoluted, no curve fitting is done. Instead, TOPAZ3D uses $g(y)$ as is and interpolates to return values for $Q$ for any $y$.

The thermal energy deposition through the thickness of the slab is given by the function $f(z)$. This function is calculated using our Lamp Model code, ${ }^{5}$ which calculates the energy deposition profile as a function of slab geometry, doping density, and lamp operating level. The profile so obtained is a spectrally-integrated thermal energy deposition profile, which we then fit to a double exponential:

$$
f(z) \propto e^{-\mu l z}+c e^{-\mu 2 z}
$$

where $\mu_{1}, \mu_{2}$, and $c$ are the fit coefficients. A plot of the energy deposition profile and the corresponding fit is shown in Fig. 3.

To describe the temporal behavior of the pump pulse, we use an analytic expression for $u(t)$. We first need to describe the temporal behavior of the electrical input power to the lamp. Since the flashlamp is a non-linear circuit element, the acutal pulse shape is described by a non-linear differential equation. ${ }^{6}$ We have found, however, that an excellent approximation to the shape is given by the function:

$$
p(t) \propto t \operatorname{Exp}\left[-(t-a)^{2} / \tau^{2}\right]
$$

where $a$ and $\tau$ are fit parameters. A plot of the electrical input power as determined from a numerical integration of the circuit equation and the approximation given by Eq. (3) is shown in Fig. 4. With the electrical input power so determined, we then need to calculate the optical output power from the flashlamp. The output power, $u(t)$, may be calculated from the following equation: ${ }^{7}$

$$
d u / d t=[\eta(u) p(t)-u(t)] / \tau_{R}
$$

where $\eta(u)$ is the instantaneous radiant efficiency of the flashlamp (corrected for arcexpansion effects), and $\tau_{R}$ is radiative recombination time of the plasma (approx. $30 \mu \mathrm{s}$ ).

" Since $\eta(u)$ is a non-linear function of $u$, Eq. (4) is likewise non-linear. ${ }^{7}$ We have found, however, that an excellent approximation is to take $\eta(u)$ to be a constant, parametric in the pulsewidth:

$$
\eta(u)=.653+2.33 \times 10^{-4} \tau_{10} \quad 200<\tau_{10}<500 \mu \mathrm{s}
$$

where $\tau_{10}$ is the full-width-tenth-max time of the electrical input power pulse. In Figure 5 we show the comparison between the numerical solution to Eq. (4) and the solution using the approximation given by Eq. (5). We see that over the time range specified, the agreement is quite good. Using Eqs. (3)-(5) an analytic expression for $u(t)$ may be obtained.

At this point in time, we do not have a good $a b$ initio calculation for the heat deposited in the laser slab. Present calculations disagree with measurements by about a factor of two; the cause of this discrepancy is currently not known. Thus, we have included a scale factor, $A$ in Eq. (1), to scale our results to experimental measurements. It should be pointed out that once this factor is determined (say, e.g., from AMPLAB measurements), then that factor is held constant for all succeeding calculations. 
The last term in Eq. (1) represents the heat deposited into the edge claddings which surround the laser glass. This term is composed of two parts:

$$
Q_{e c}=Q_{e c, p u m p}+Q_{e c, A S E}
$$

where $Q_{\text {ec,pump,ASE }}$ represents the heat deposited into the edge claddings by the pump, ASE respectively. The source term is broken up in such a manner because the time dependence of the two parts is different. For $Q_{\text {ec,pump }}$ the time dependence is just $u(t)$, described above. For $Q_{\text {ec.ASE }}$ the time dependence is different due the fact that the peak of the ASE pulse occurs at the time of peak gain, not at the time of peak pump power. The relationship between the output power from the flashlamp and the stored energy density, $\rho(t)$, is given in Ref. 7. Once $\rho(t)$ is determined, it may be shown that the time dependence of the ASE, $\phi(t)$ may be written as: ${ }^{8}$

$$
\phi(t) \propto \rho(t)[a+b\{\operatorname{Exp}[\rho(t)]-1\}]
$$

where $a$ and $b$ are constants. With Eq. (7) describing the time dependence of the ASE, we then have, for example, for the edge cladding at $x=$ const.:

$$
Q_{e c, A S E} \propto \phi(t) \beta \Gamma \mathrm{e}^{-\beta x}
$$

where $\beta$ is the edge-cladding absorption coefficient, and $\Gamma$ is the incident fluence. At present, we do not have an accurate $a b$ initio calculation of the ASE fluence on the edge cladding. However, based on measurements with the Beamlet laser, we estimate a fluence of $4 \mathrm{~J} / \mathrm{cm}^{2}$ for ASE and another $2.5 \mathrm{~J} / \mathrm{cm}^{2}$ due to the pump light.

Equations (1)-( 8) are used in TOPAZ3D to determine the temperature distribution in the laser slab. Due to the shortness of the pump pulse (a few hundred $\mu \mathrm{sec}$ ), adiabatic boundary conditions are used on all faces of the slab. The result of this calculation is used in the code NIKE3D to calculate the displacements and stresses, as described in the next section.

\subsection{Determination of displacements and stresses}

As mentioned above, we use the temperature distribution in the slab to calculate the displacements and stresses. Since the displacements are very small (on the order of $1 \mu$ ), we are in the linear elastic regime and the problem is a standard one in thermoelasticity. Consequently, we use a thermo-elastic material model and specify Poisson's Ratio, Young's Modulus and thermal expansion coefficient. ${ }^{9}$ These parameters are taken to be constants, independent of temperature, insofar as the maximum temperature rise is on the order of one degree Celsius.

At this point in the calculation, the mechanical boundary conditions for the slab are specified. In reality, the slab sits on a Marcel spring - a sinusoidally varying metal strip. While the capability exists to model the spring as it actually exists, we have found that a satisfactory substitution is to have the slab sit on a region of metal one element thick. The nodes in the glass material are joined to the nodes in the metal so no slipping can occur. The bottom of the metal region is simply supported and we also fix the displacements at two additional corners to eliminate rigid-body motion. The rest of the slab surfaces are assumed to be free. 
NIKE3D calculates the displacements and stresses as a function of time during the course of the pump pulse. Typically, the code is run up to the time of peak gain as the wavefront distortion at that time is what is usually requested. However, it is a simple matter to run the code for times longer than the time of peak gain in order to compare with experiments.

\subsection{Determination of OPDs}

The last part of the calculation involves computing the OPDs through the laser slab. For this, we use our in-house code OPL. The optical path length of a ray through the slab may be written as:

$$
\mathrm{OPL}=\int n(x, y, z) d s
$$

where $n$ is the (spatially-varying) refractive index and $s$ is the distance along the ray path. There are two main sources of OPD in the laser slab: 1) Variations in path length caused by mechanical motion of the slab, and 2) Variations in path length caused by refractive index changes.

The variations in path length caused by mechanic al motion of the slab are caused by the spatially-varying displacements calculated in NIKE3D; i.e. a point $x, y, z$ on the slab gets translated to:

$$
\begin{aligned}
x & \rightarrow x+u(x, y, z, t) \\
y & \rightarrow y+v(x, y, z, t) \\
z & \rightarrow z+w(x, y, z, t)
\end{aligned}
$$

We take two effects into account to calculate the spatially-varying refractive index: 1) The variation of refractive index with temperature, and 2) the variation of refractive index with stress (stress-optic effect), i.e.:

$$
n(x, y, z)=n_{0}+(d n / d T) \Delta T(x, y, z)+(d n / d \sigma) \Delta \sigma(x, y, z)
$$

where $n_{0}$ is the isotropic refractive index, $d n / d T$ denotes the change of refractive index with temperature, and we have symbolically written the change in refractive index due to stress as $(d n / d \sigma)$. Note that in general, $\Delta T$ and $\Delta \sigma$ are functions of time. However, for the purposes of calculating the OPD, we select one point in time for the calculation. This is permissible since the time duration of the laser pulse is at most $20 \mathrm{~ns}$. On this time scale, all thermal and mechanical motion is frozen.

The sequence of events in calculating the OPD is as follows. The OPL code reads in the finite-element geometry from the NIKE3D plot file. We then go through the mesh and break up each finite-element "brick" into six four-node tetrahedra and generate a connectivity matrix for these tetrahedra. We then use Eq. (11) to calculate the refractive index at each node in the mesh. Within each tetrahedra, we linearize the refractive index:

$$
n(x, y, z)=a+b x+c y+d z
$$


The four unknowns in Eq. (12) are uniquely determined by the values of the refractive index at the four nodes of a given tetrahedron. With the refractive index linearized as in Eq. (12), we can then analytically solve the Eikonal equation ${ }^{10}$ for the ray path within a tetrahedron:

$$
\frac{d}{d s}\left(n \frac{d \vec{r}}{d s}\right)=\nabla n
$$

where $s$ is the distance along the ray path and $\mathbf{r}$ is the position vector of the ray. The connectivity matrix helps us determine which tetrahedron the ray will enter and consequently at which nodes to evaluate the refractive index in order to calculate the unknowns in Eq. (12). We then track the ray as it propagates through all the tetrahedra, all the while accumulating the distance that the ray propagates.

In addition to calculating the OPD for the ray, we can also calculate the depolarization a ray experiences as it propagates through the optic. We do this by assuming each tetrahedron acts as a linear retarder and calculate the Jones matrix ${ }^{11}$ for each tetrahedron. The final amount of retardation (and hence depolarization) is given by the product of all the individual Jones matricies for a given ray path.

A typical result from this calculation is shown in Fig. 6. The calculation was performed for the AMPLAB amplifer in the Diamond configuration. In Fig. 6 (a) we show the OPD for all effects (displacement, temperature, and stress) combined. It is often useful to examine each component indivudually and this is done in Figs. 6(b), (c), and (d). In Fig. 6(b) we show the contribution to the OPD due solely to mechanical deformation. Comparison with Fig. 6(a) shows that for AMPLAB, the wavefront distortion is due mainly to the mechanical deformation of the slab. In Figs. 6(c) and (d) we show the contriubtion to the OPD from temperature and stress effects on the refractive index. As may be seen, these effects play a relatively minor role in determining the overall OPD. Finally, in Fig. 6(e) we show the P to S depolarization. As expected, the greatest amount of depolarization occurs in the comers, where the two pieces of edge cladding meet; it is there where the greatest amount of stress occurs. Nevertheless, the overall amount of depolarization is small, well within its specification of $.05 \%$ averaged over the aperture.

\subsection{Error analysis}

In this section, we will estimate the error in our calculation of the OPD. For the amplifier conditions considered in this report, the dominant contribution to the OPD is the mechanical deformation of the laser slab ( $c f$. Figs. 6(a) and (b)). Consequently, it makes sense to closely analyze the uncertainties associated with mechanical motion.

For simplicity, assume the laser slab is a simply-supported thin plate, with the thin dimension along the $z$-axis. We will neglect any time dependence in this analysis. It may be shown that the equation for $w$, the displacement in the $z$-direction, is given by: ${ }^{12}$

$$
\nabla^{2} w(x, y)=-\left(\frac{M_{T}(x, y)}{1-v}\right)
$$

where the thermal moment, defined as: ${ }^{12}$ 


$$
M_{T}(x, y)=\alpha E \int_{0}^{h}(z-h / 2) T(x, y, z) d z
$$

is the source function for the displacement. In Eqs. $(14,15) v$ is Poisson's Ration, $\alpha$ is the thermal expansion coefficient, and $E$ is Young's modulus.

On the time scales of interest, one may neglect diffusion. Consequently, $T(x, y, z)$ $\propto Q(x, y, z)$. If one substitutes Eq. (1) into Eq. (14) using Eq. (2), one finds that the thermal moment is proportional to the difference in pump profiles, i.e. $\mathrm{M}_{\mathrm{T}} \propto \mathrm{g}_{0}(y)-\mathrm{g}_{\mathrm{h}}(y)$. Consequently, small uncertainties in the values of $g_{0}$ and $g_{h}$ can lead to large uncertainties in the thermal moment, and hence the amount of deformation. Since the steering of the laser beam is driven by the curvature of the laser slab, it follows that the phase front, which is the integral of the beamsteering, is proportional to the gradient of the displacement, or the integral of the thermal moment.

With our 2-D ray-trace code, we can match the gain profile across the aperture to within $1 \%$. Because of ASE within the laser slab, we can vary the pump profile by $2 \%$ and still be within $1 \%$ in the gain coefficient. Consequently, if we take the pump profiles shown in Fig. 1, and assume a worse-case uncertainty of $2 \%$, it can be shown that the variation in the peak-to-valley value for the phase front can be as much as $\pm 15 \%$.

\section{Comparison with AMPLAB experiments}

In this section, we present comparisons of experiments performed in AMPLAB with the model described in the previous section. Unless otherwise mentioned, all comparisons are done at the time of peak gain at an explosion fraction of 0.2. As shown in Fig. 6, the OPD is calculated over the entire aperture. However to facilitate making comparison with the data, we shall show horizontal lineouts of calculations and experiments. These lineouts were taken at the vertical midplane of the aperture.

In Figs. 7(a) and (b), we show the comparison between the calculated and measured phase front (essentially the negative of the OPD) for the Diamond and X configurations in AMPLAB. Also indicated on the experimental curve is a typical value for the error in the measurement. As may be seen, there is excellent agreement in both configurations over the entire aperture.

In Figs. 7(c) and (d), we show the calculated and measured phase front for the three-slab-long and interior configurations respectively. As indicated on the graph, the data for the interior configuration was interpolated from the measured Diamond, X, and 3-long data using the following algorithm:

$$
\phi_{i}=\phi_{3} / 2-\phi_{d}-\phi_{x}
$$

where $\phi_{3}$ is the measured 3-slab-long phase in waves and all other phases are in waves/slab/pass. As can be seen from the figure, there is excellent agreement between calculation and measurement over the entire aperture.

Another check on the model is to calculate the wavefront distortion at times other than the time of peak gain. The resuls of these calculations, and comparison with the measurement, is shown in Figs. 8(a)-(f) (Fig. 8(b) repeated for ease of comparison). In 
these experiments, we measured the prompt wavefront distortion at $100,200,300$, and $500 \mu \mathrm{s}$ after the time of peak gain. As may be seen, the amount of wavefront distortion continues to increase after the time of peak gain up to $500 \mu \mathrm{s}$. In fact, the peak-to-valley value of the wavefront distortion is about $3 x$ greater at $t_{\text {peak gain }}+500 \mu$ s than at $t_{\text {peak gain. }}$. The agreement of the model with the measurement is excellent, matching both the mägnitude and the shape of the wavefront at all times.

\section{Summary and conclusions}

We have presented the results of detailed analysis and modeling of the AMPLAB data. The table below summarizes our analysis of the AMPLAB data:

$\begin{array}{ccc}\text { Configuration } & \begin{array}{c}\text { Meas. } \phi \text { - horiz. } \\ \text { component } \\ \text { (waves/slab/pass) }\end{array} & \begin{array}{c}\text { Calc. } \phi \text { - horiz. } \\ \text { (wave/slab/pass- } \\ \text { LG-770) }\end{array} \\ \text { Diamond } & .22 \pm .03 & .21 \pm .03 \\ \mathrm{X} & .29 \pm .03 & .27 \pm .04 \\ \text { Interior } & .18 \pm .03 & .16 \pm .02\end{array}$

We have also presented a description of our prompt pump-induced wavefront model. This model calculates the wavefront distortion due to mechanical deformation and refractive index changes due to temperature and stress. We have benchmarked the code against AMPLAB measurements and will be using it to predict the wavefront distortion for the NIF amplifiers.

\section{References}

[1] A. Shapiro, "TOPAZ3D - A 3-D Finite Element Heat Transfer Code," UCID-20484, LLNL, Livermore, CA., Aug. 1985.

[2] B. N. Maker, "NIKE3D - A Nonlinear, Implicit 3-D Finite Element Code for Solid and Structural Mechanics," UCRL-MA-105268, Rev. 1, LLNL, Livermore, CA., April 1995.

[3] S. Doss and R. Gelinas, "Mathematics and Physics of the BREW Code," in Laser Program Annual Report, UCRL-50021-86, LLNL, Livermore, CA., p. 7-132, (1986).

[4] [2] G. LeTouzé, Olivier Cabourdin, J.F. Mengue, M. Rotter and K. Jancaitis. "Shaped reflectors for Pump Cavities," 2nd Annual Conf. Solid State Lasers for Application to ICF. Limeil, France 1996

[5] K. Jancaitis, "Flashlamp Modeling," in Laser Program Annual Report, UCRL 5002186, LLNL, Livermore, CA. p.6-3, (1986).

[6] J.P. Markiewicz and J.L. Emmett, "Design of Flashlamp Driving Circuits,", IEEE J.Q.E., QE-2, p. 707, (1966).

[7] H.T. Powell, A.C. Erlandson, K.S. Jancaitis, and J.E. Murray, "Flashlamp Pumping of Nd:Glass Disk Amplifiers," SPIE 1277, p. 103, (1990). 
[8] K.S. Jancaitis, "Disk Amplifier Performance Model," in Laser Program Annual Report, UCRL-LR-105820-88/89, LLNL, Livermore, CA., p. 6-12, (1993).

[9] S.E. Stokowski, R.A. Saroyan, and M.J. Weber, "Laser Glass - Nd-doped Glass Spectroscopic and Physical Properties, V1," M-095, Rev. 2, V. 1, LLNL, Livermore, CA., (1981).

[10] M. Born and E. Wolf, "Principles of Optics, 6 ${ }^{\text {th }}$ Ed.", p. 122, Pergamon Press, New York, (1980).

[11] R.M.A. Azzam and N.M. Bashara, "Ellipsometry and Polarized Light," p. 488, North-Holland, Amsterdam, (1987).

[12] B. Boley, and J. Weiner, "Theory of Thermal Stresses," Ch. 12, J. Wiley \& Sons, New York, (1960). 


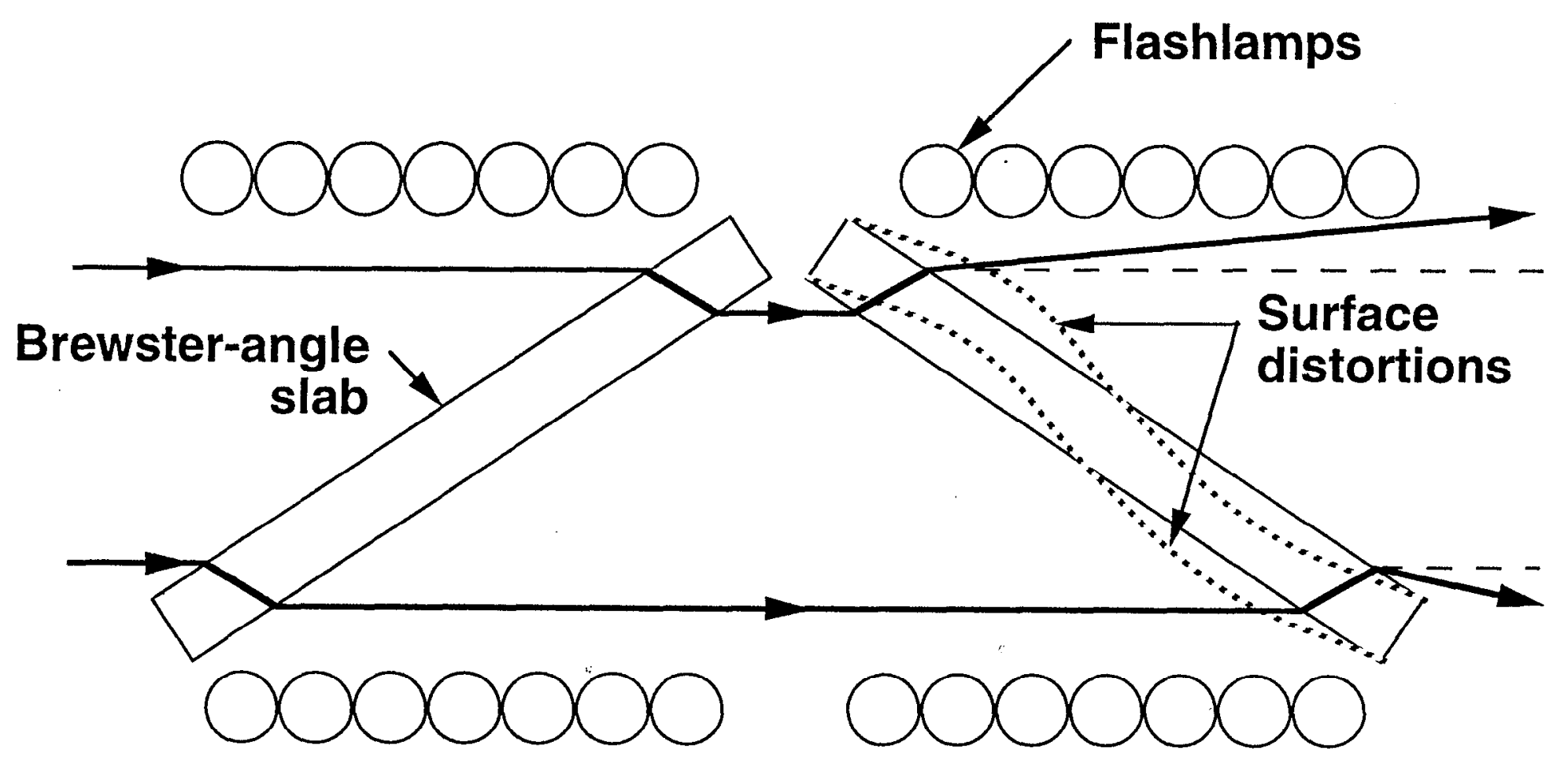

Fig. 1 Plan view of multi-segment amplifer showing geometry of Brewster-angle laser slabs. Surface distortions (greatly exaggerated) caused by uneven pumping. 


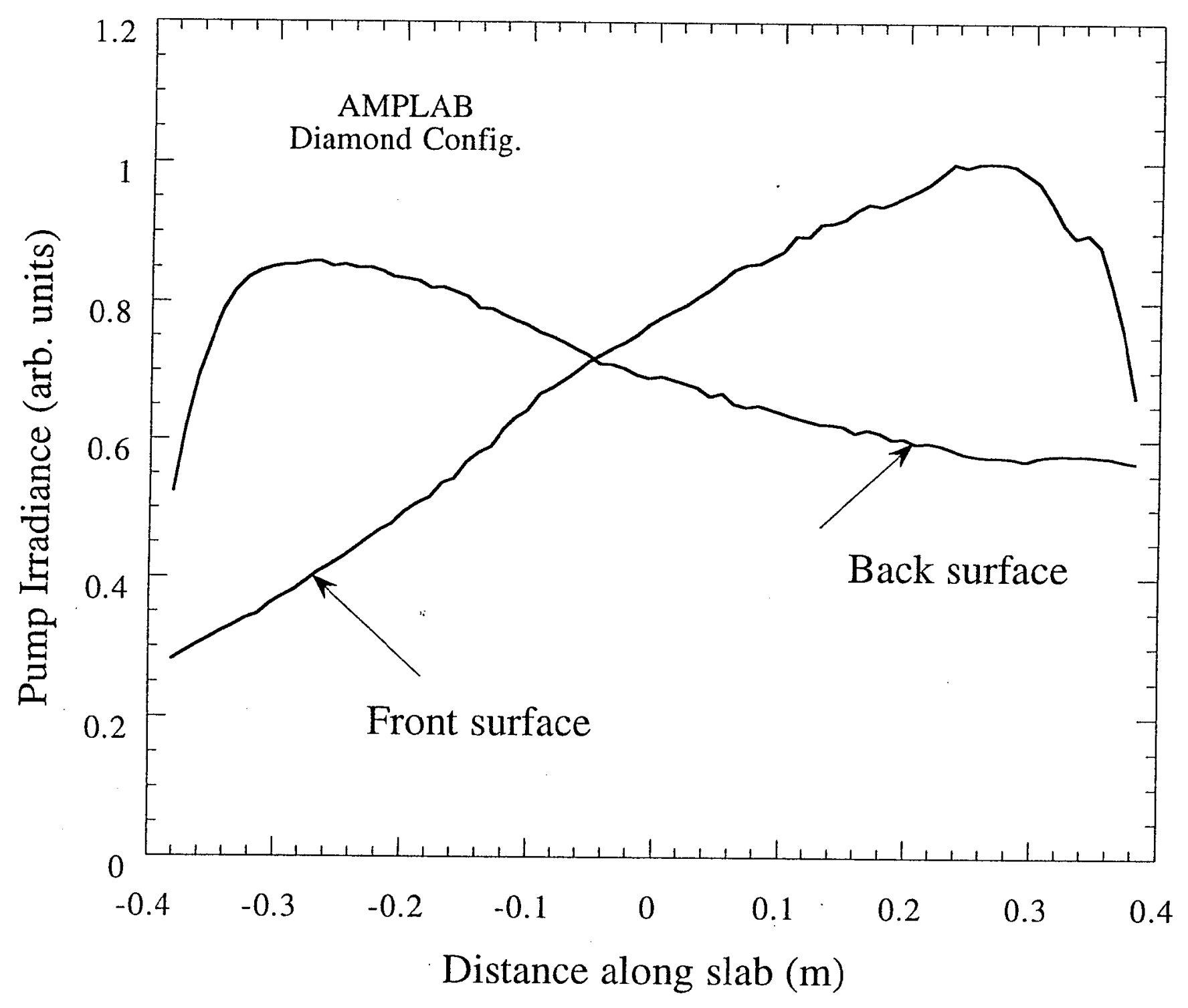

Fig. 2: Pump irradiance profiles - AMPLAB Diamond configuration 


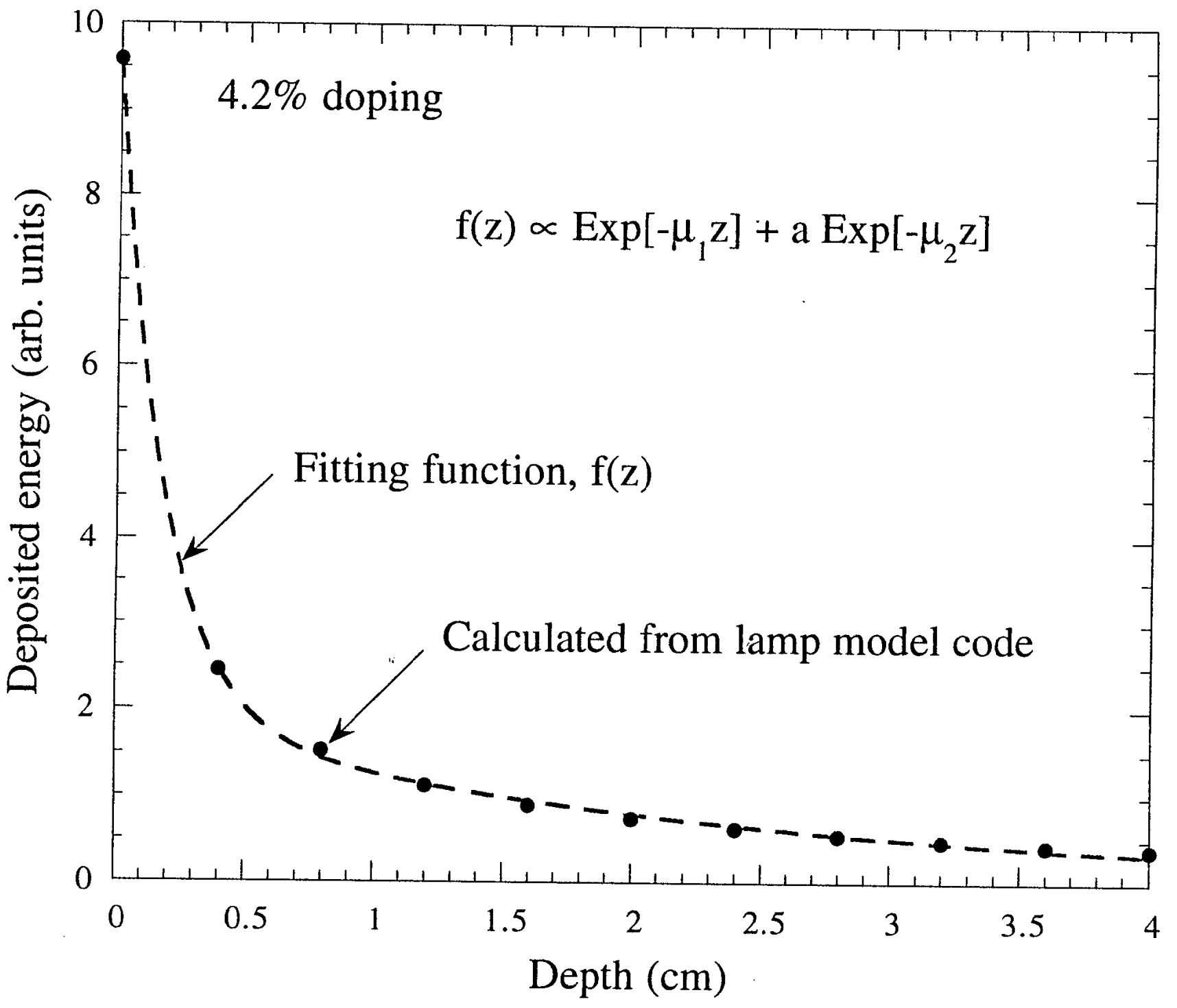

Fig. 3: Energy deposition profile through slab $(\bullet)$ and fit (- - ) 


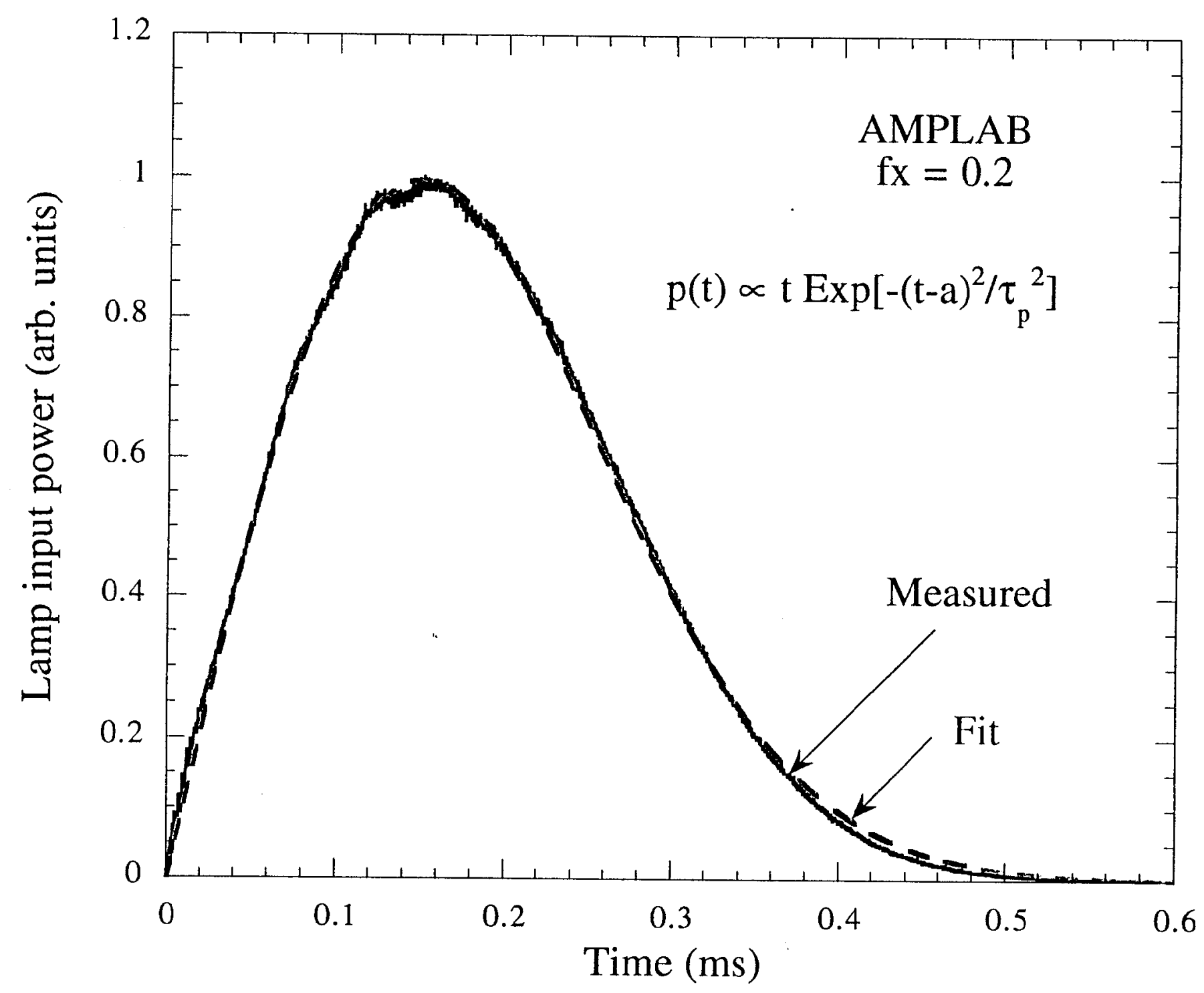

Fig. 4: Measured electrical input power to lamp ( - ) and fit (- - ) 

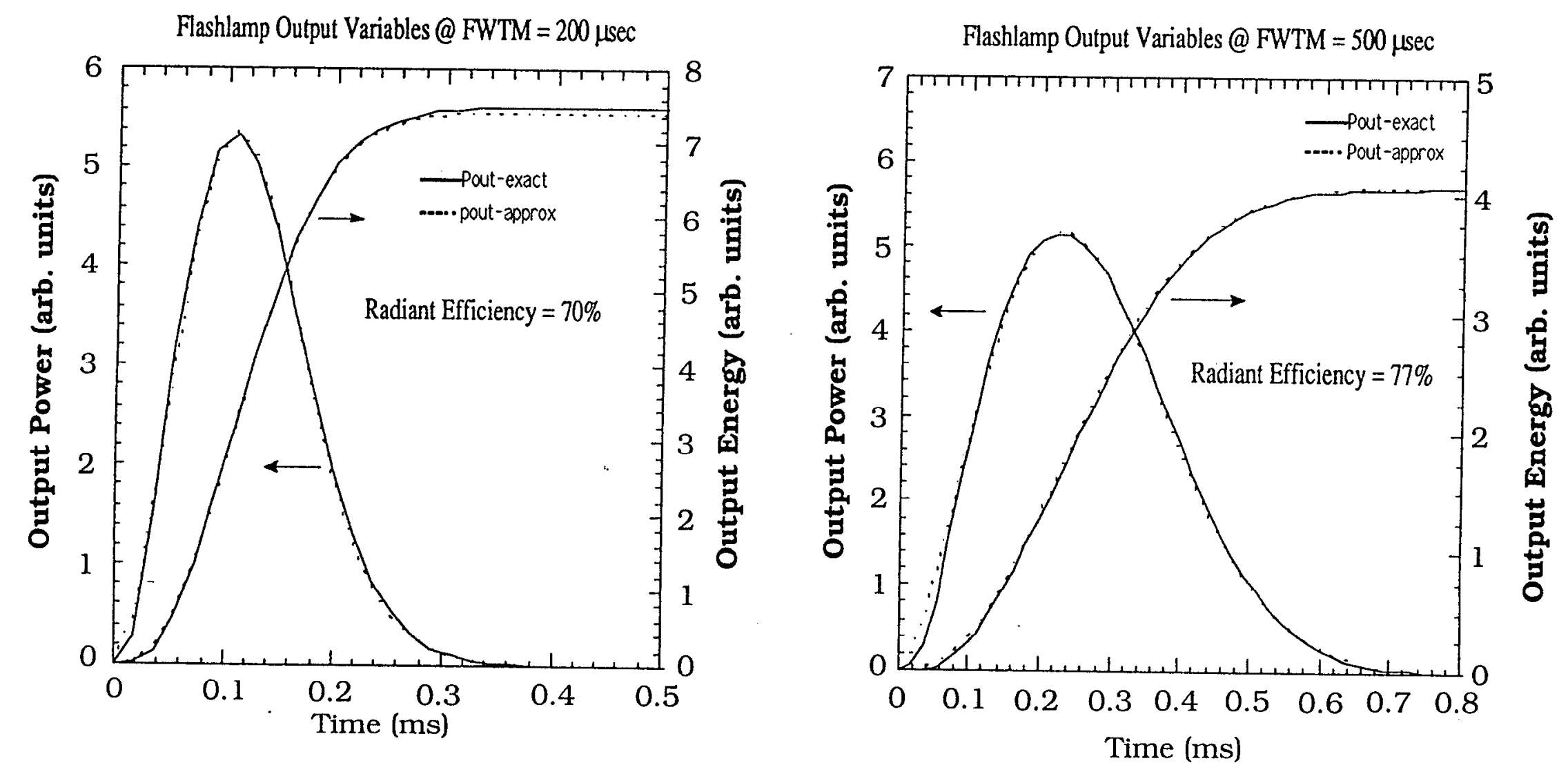

Fig. 5: Comparison of exact and approximate solutions to flashlamp output equation 


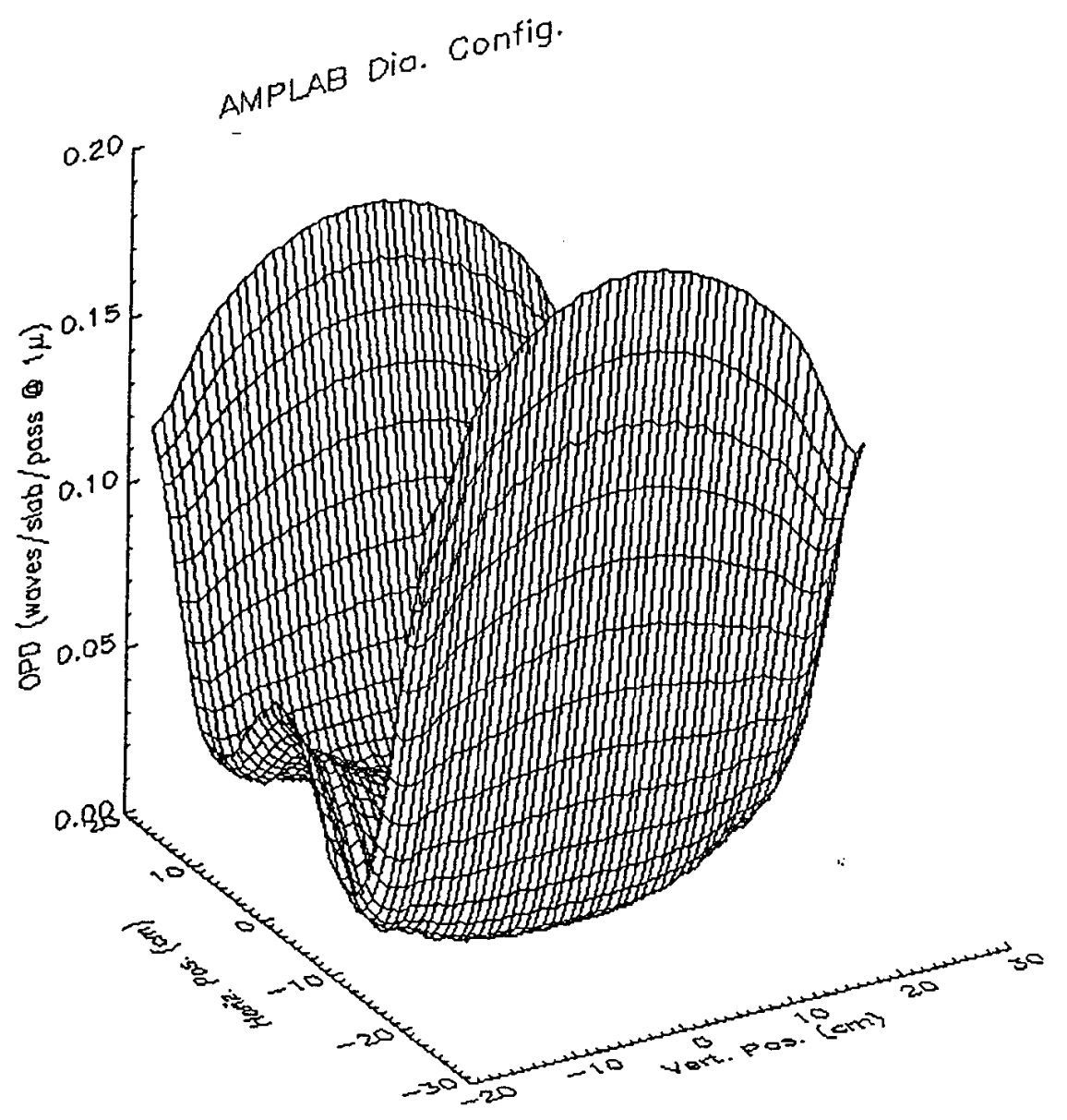

(a)

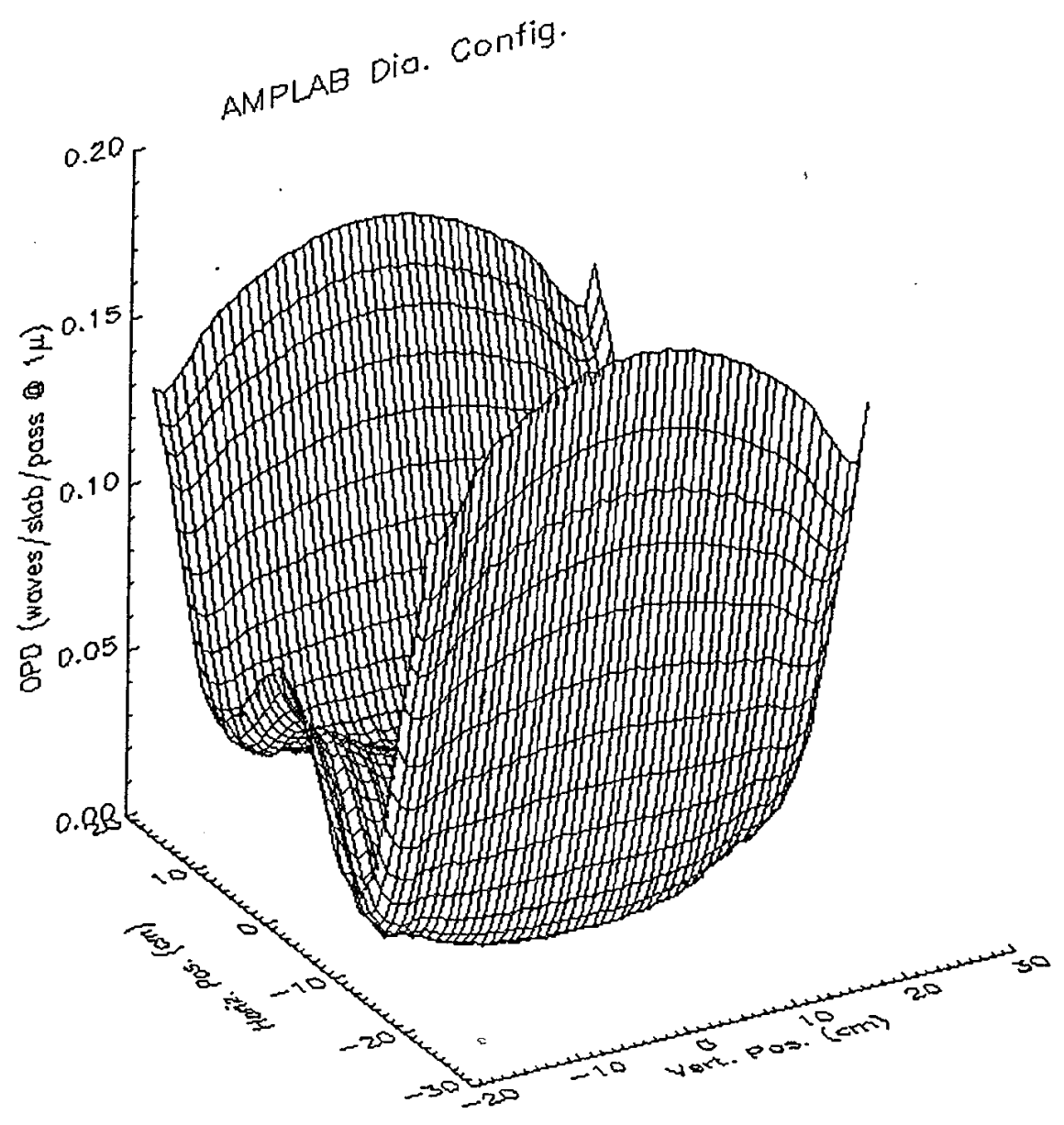

(b)

Fig. 6: OPD calculated for the AMPLAB Diamond configuration, $f_{x}=0.2$ :

(a) All Effects, (b) Displacement effects only 


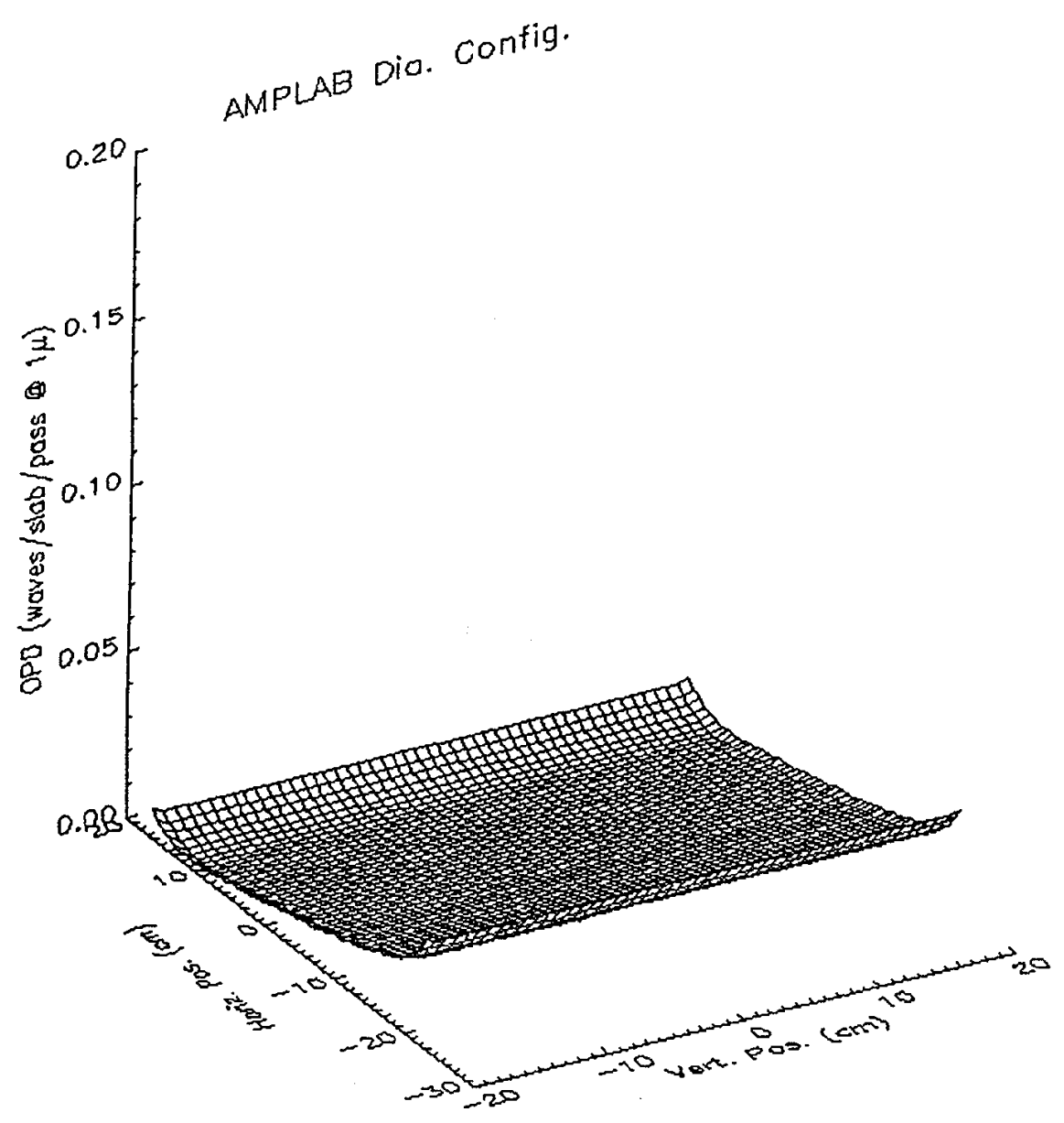

(c)

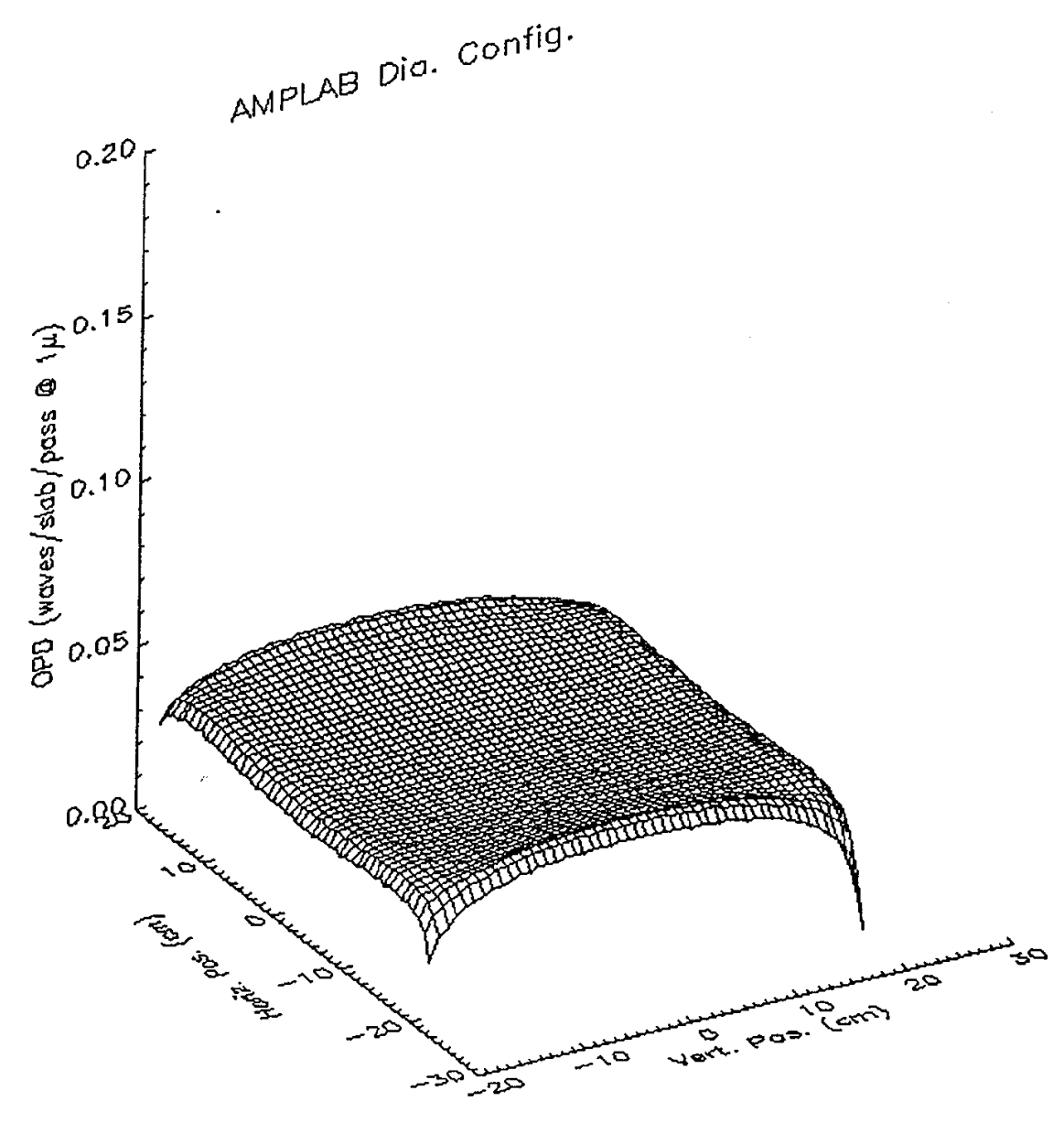

(d)

Fig. 6(cont'd): OPD calculated for the AMPLAB Diamond configuration, $f_{x}=0.2$ :

(c) dn/dT effects only, (d) Stess effects only 


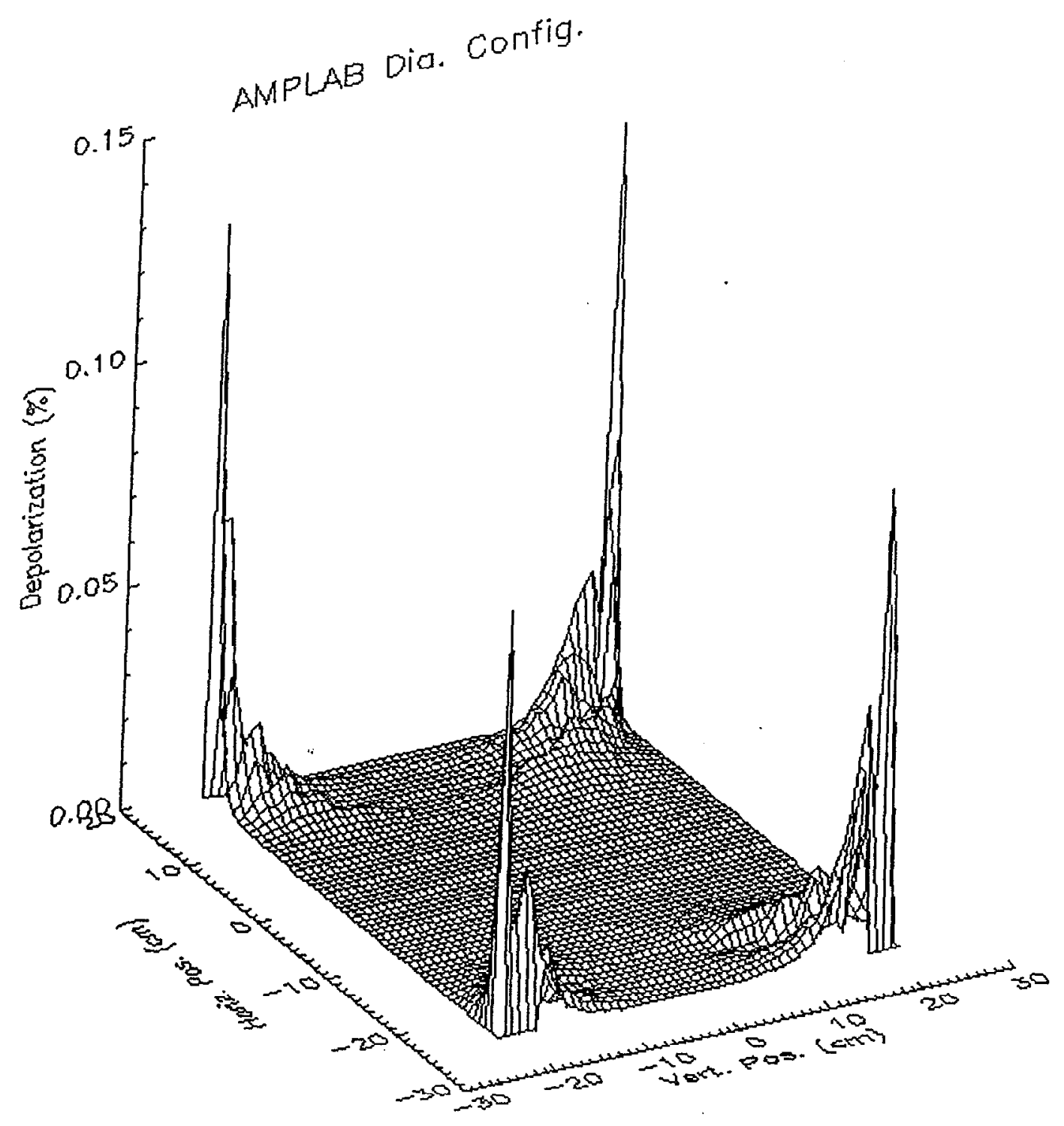

(e)

Fig. 6(cont'd): (e)-Depolarization calculated for the AMPLAB Diamond configuration, $f_{x}=0.2$ 


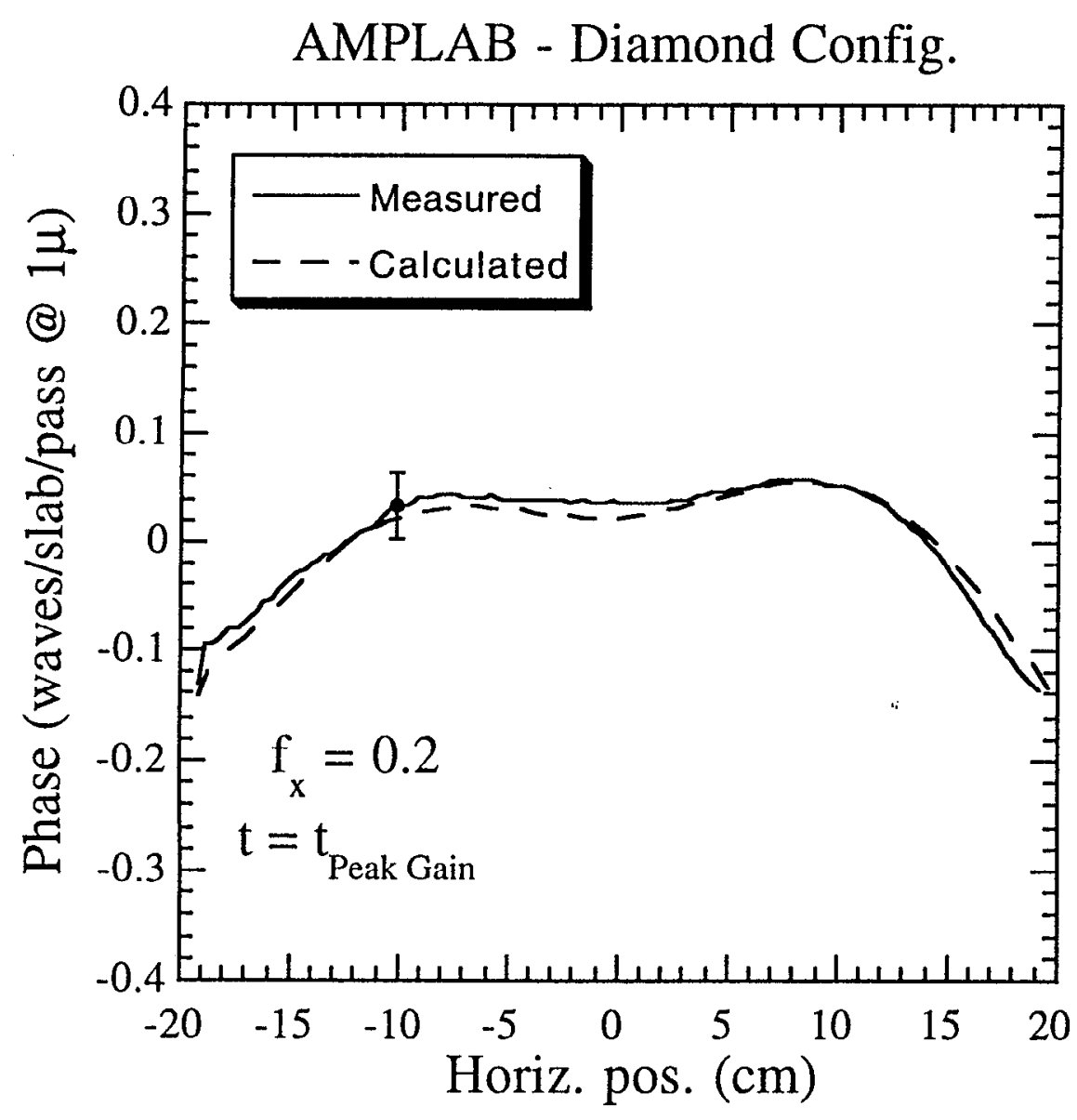

(a)

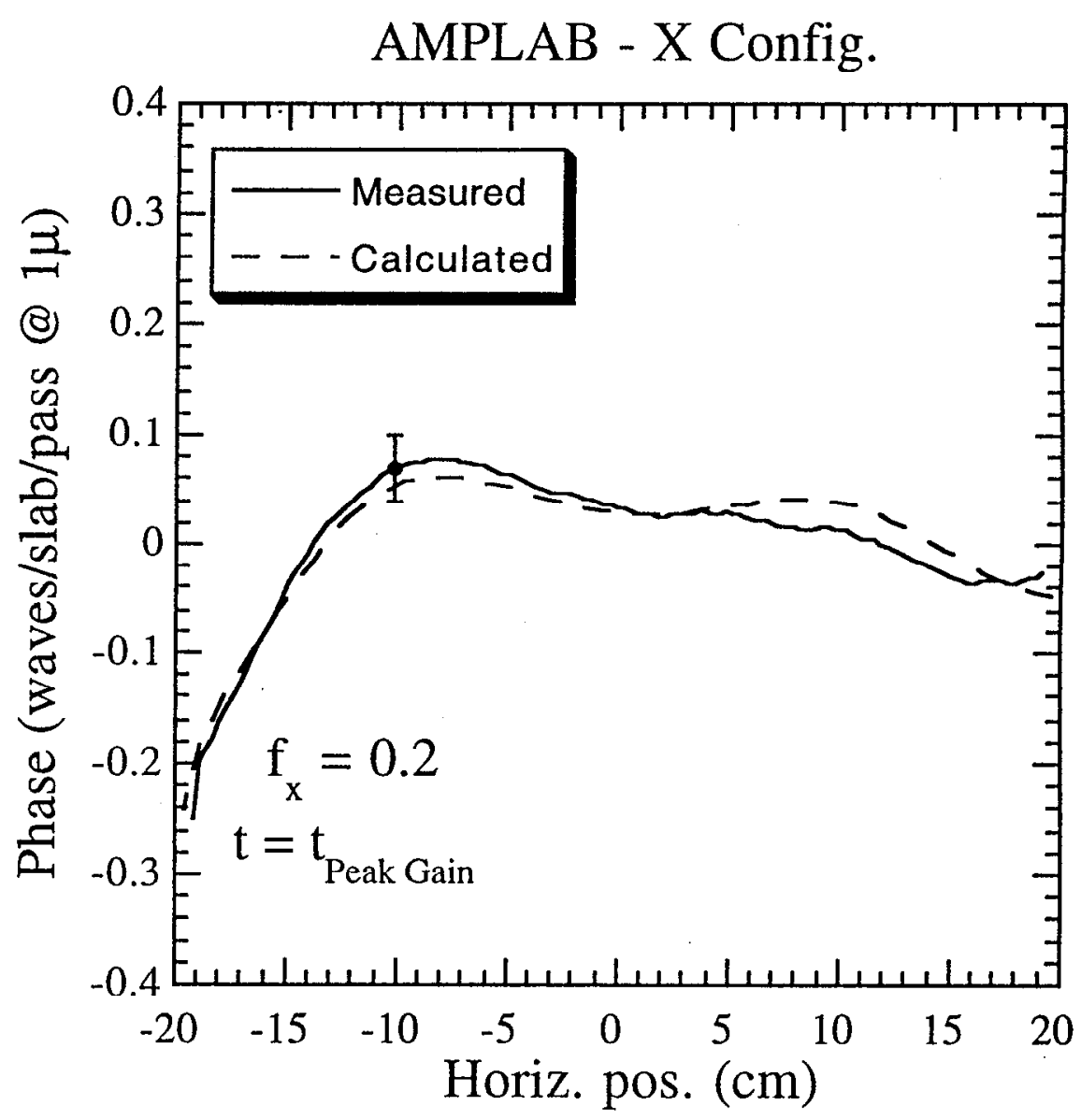

(b)

Fig. 7: Measured and calculated wavefront, horizontal component - AMPLAB:

(a) Diamond configuration, (b) $\mathrm{X}$ configuration. Error bar shows typical error for measurement. 


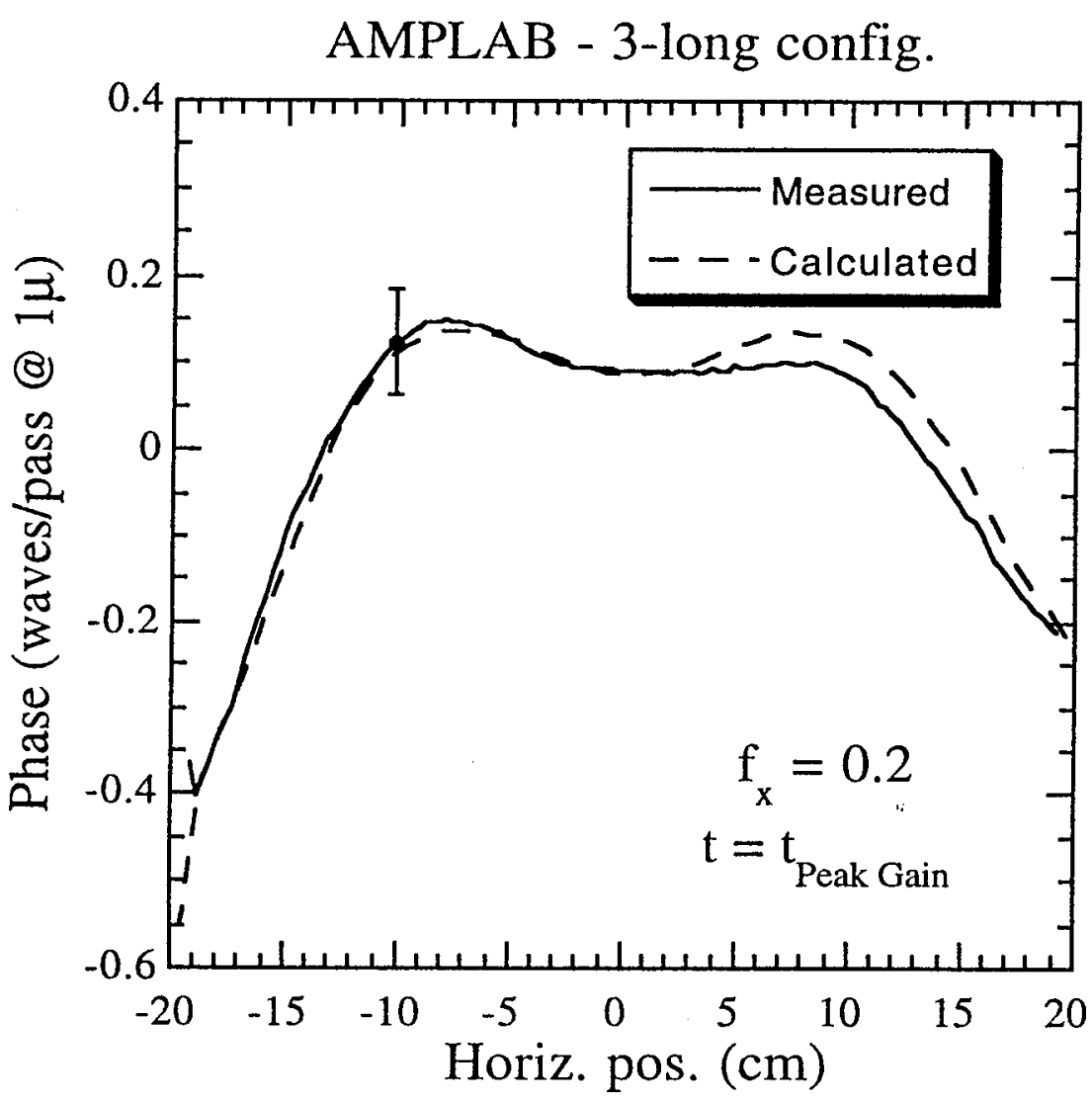

(c)

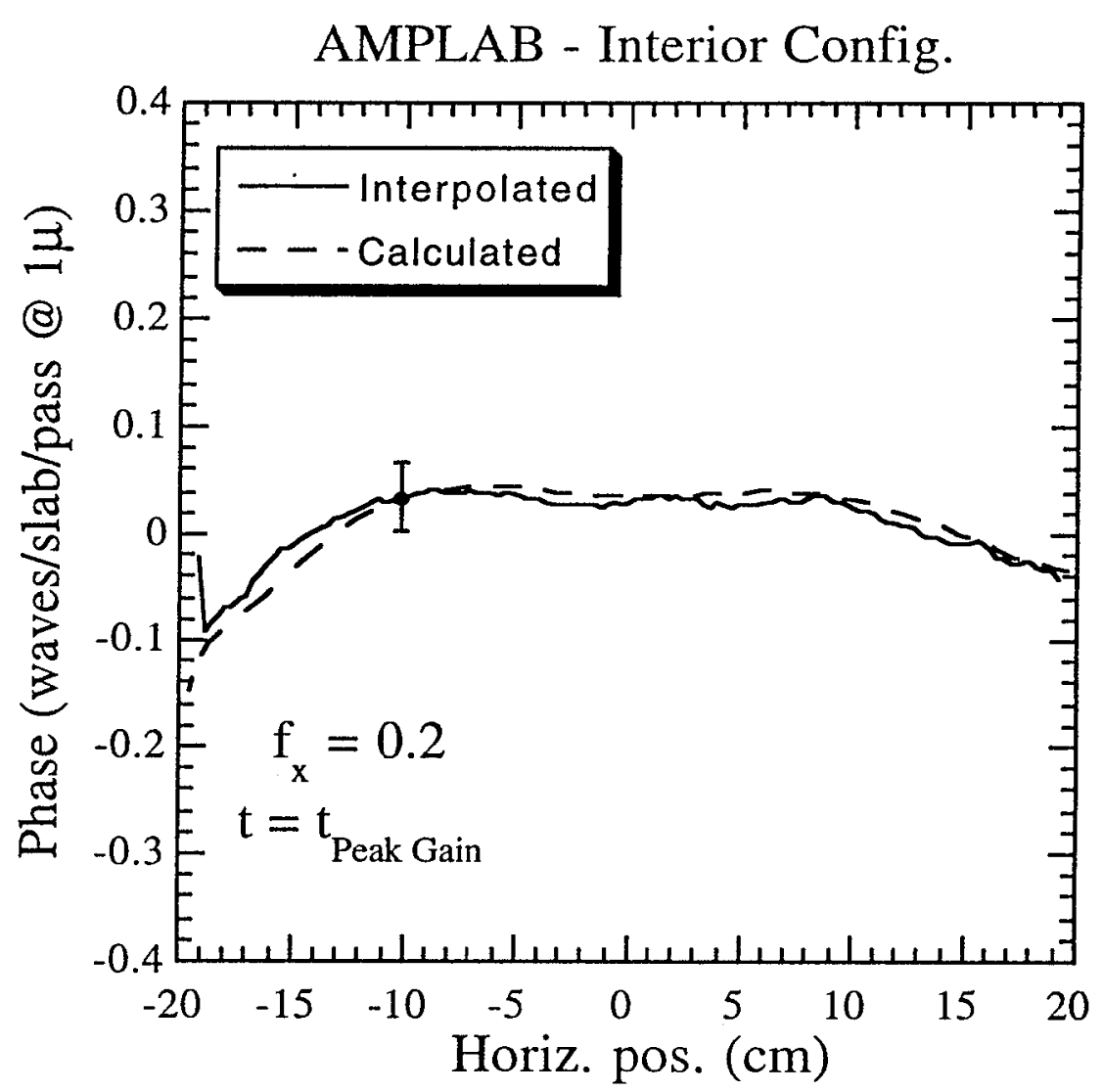

(d)

Fig. 7(cont'd): Measured and calculated wavefront, horizontal component - AMPLAB:

(c) 3-slab-long configuration, (d) Interior configuration - interpolated from 3-long, diamond, and $\mathrm{X}$ results. 


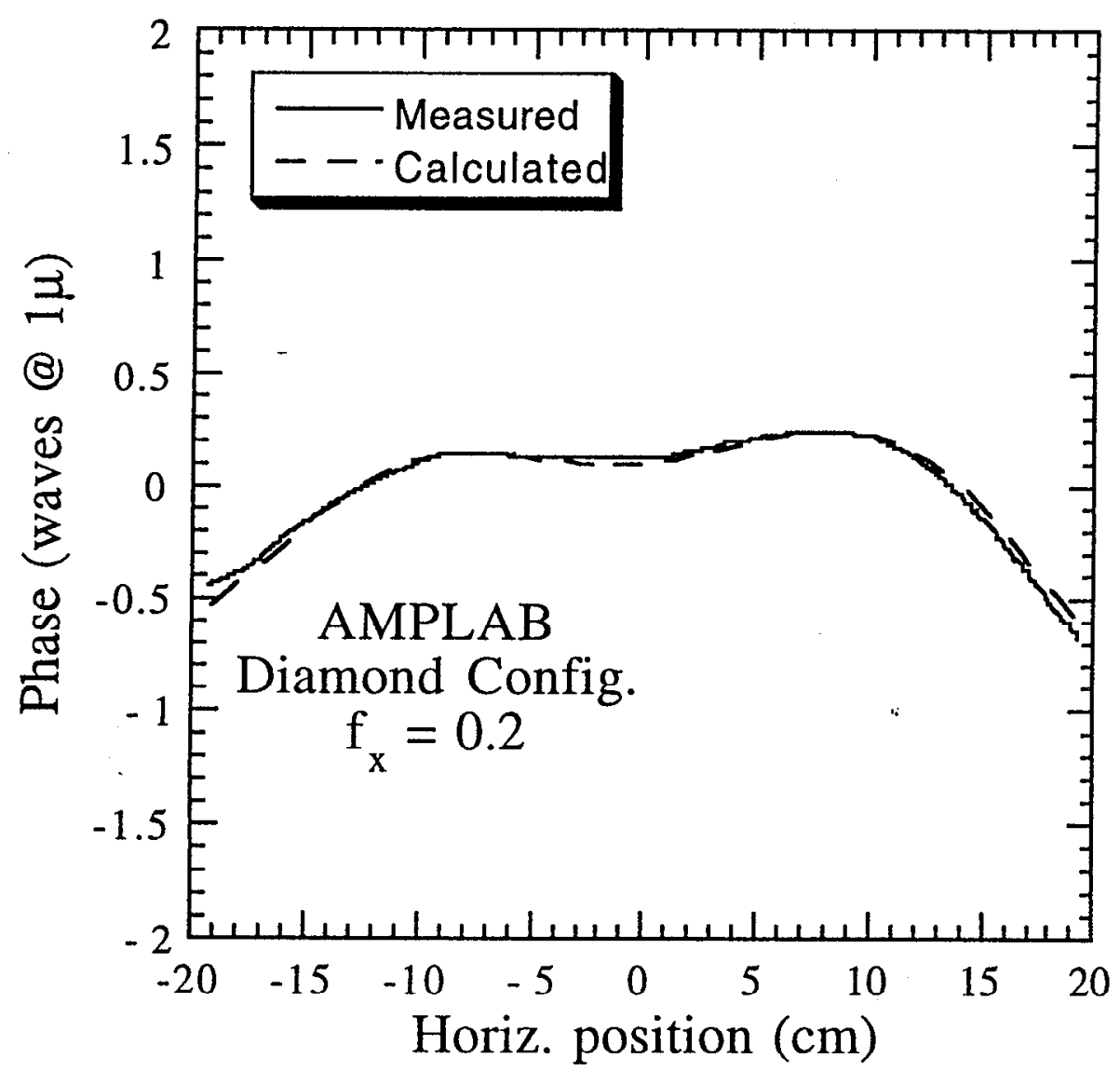

(a)

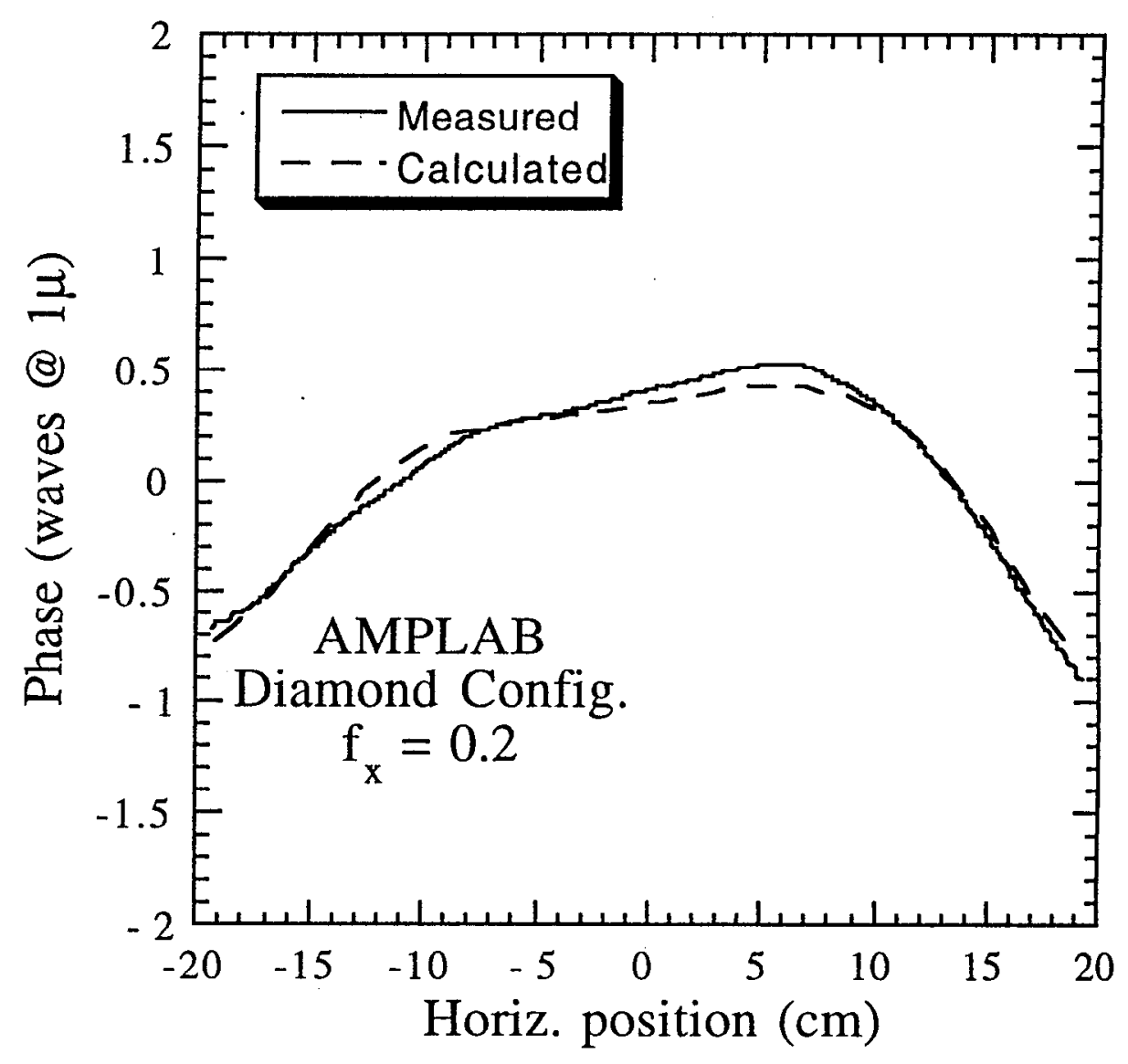

(b)

Fig. 8: Measured and calculated wavefront, horizontal component - AMPLAB,

Diamond configuration: measurements taken at (a) $t=t_{\text {peak gain }}$,

(b) $\mathrm{t}=\mathrm{t}_{\text {peak gain }}+0.1 \mathrm{~ms}$ 


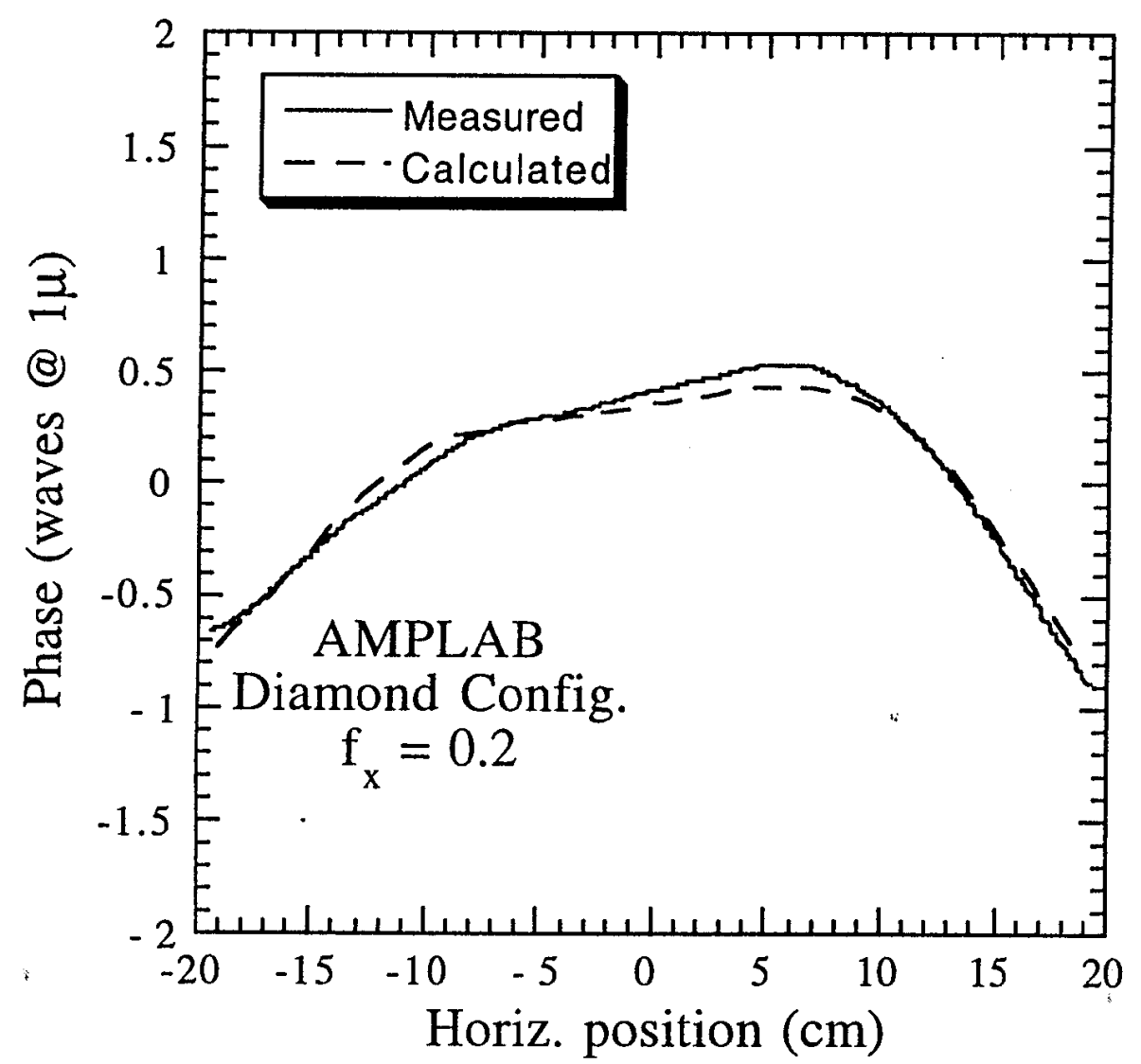

(c)

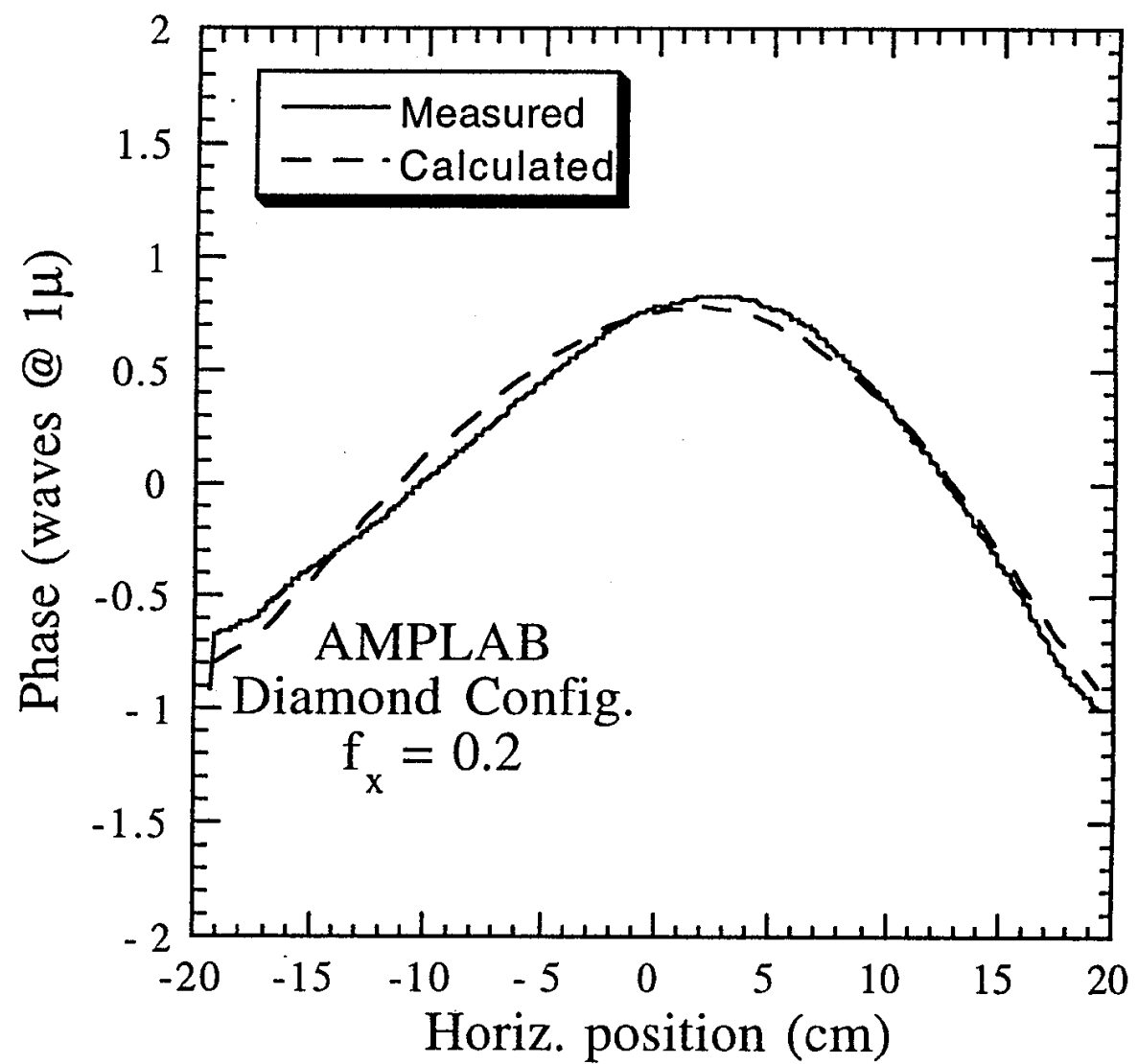

(d)

Fig. 8(cont'd): Measured and calculated wavefront, horizontal component - AMPLAB, Diamond configuration: measurements taken at (c) $t=t_{\text {peak gain }}+0.1 \mathrm{~ms}$ (d) $\mathrm{t}=\mathrm{t}_{\text {peak gain }}+0.2 \mathrm{~ms}$ 


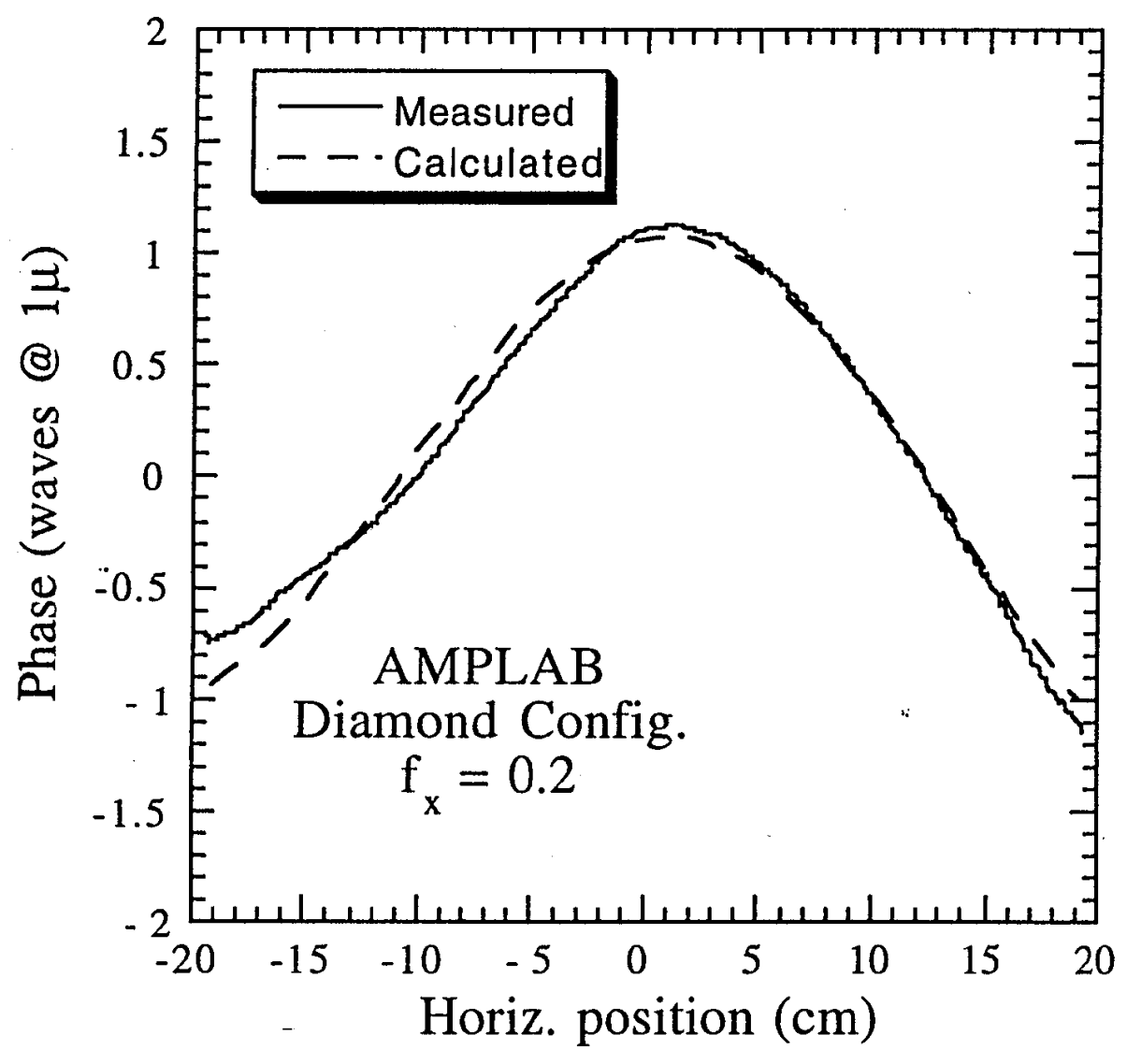

(e)

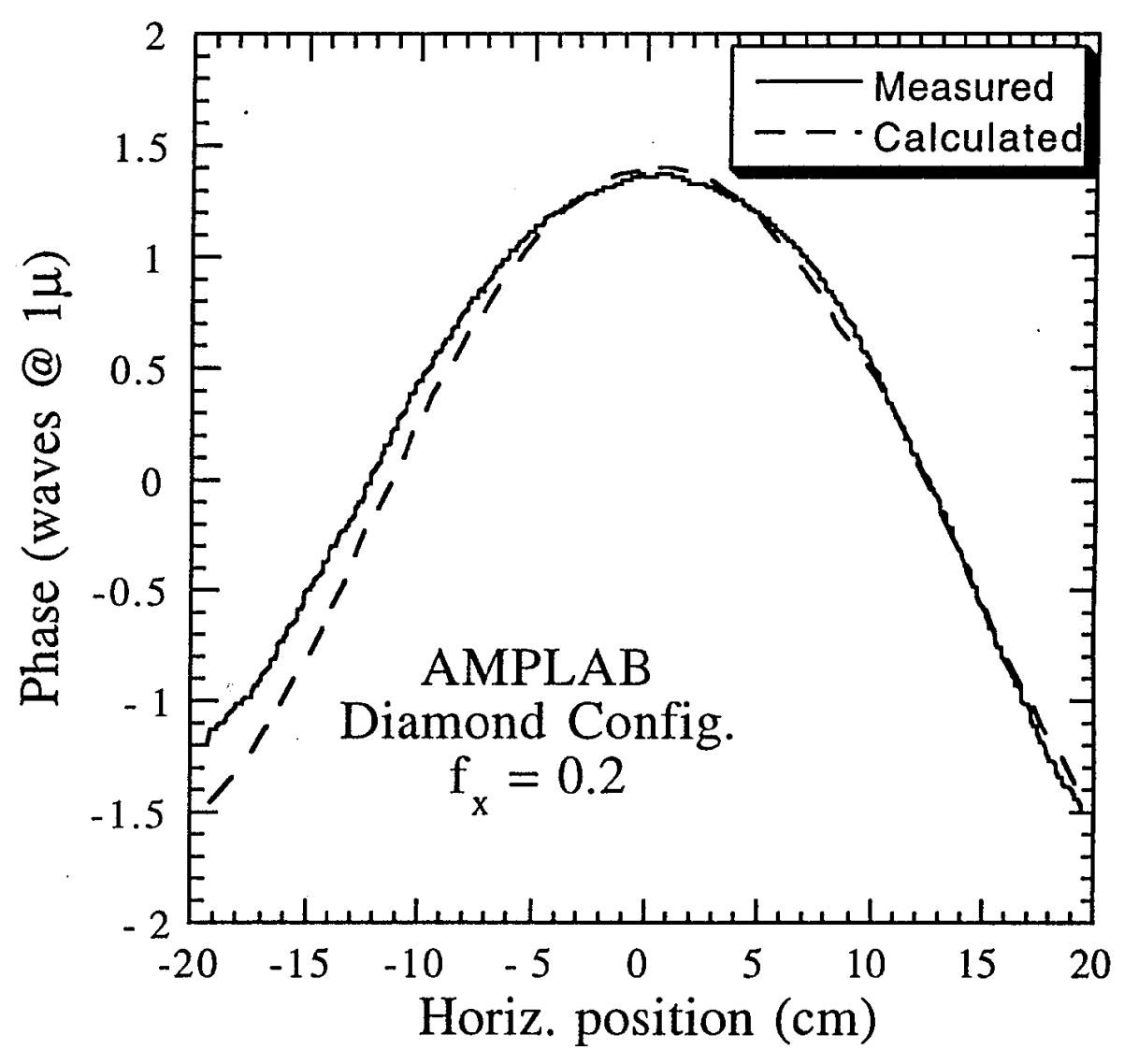

(f)

Fig. 8(cont'd): Measured and calculated wavefront, horizontal component - AMPLAB, Diamond configuration: measurements taken at (c) $t=t_{\text {peak gain }}+0.3 \mathrm{~ms}$ (d) $\mathrm{t}=\mathrm{t}_{\text {peak gain }}+0.5 \mathrm{~ms}$ 